\title{
Improved Enhanced Distributed Energy Efficient Clustering (iE-DEEC) Scheme for heterogeneous Wireless Sensor Network
}

\author{
F. Jibreel \\ Department of Computer Science \\ Tamale Technical University \\ Tamale, Ghana
}

\section{ABSTRACT}

Wireless Sensor Networks (WSNs) contain thousands of sensor nodes which are capable of conveying sensed and captured data from any deployment area to another well-resourced node called the Base station for analysis. Due to the ability of the sensor nodes to relay data from one place to the other, they are found applicable in several areas such as environmental monitoring, military surveillance and home intelligence. The operation of the heterogeneous routing algorithm, E-DEEC (Enhanced Distributed Energy-Efficient Clustering) for heterogeneous networks has been observed carefully. The E-DEEC scheme improved the DEEC algorithm by introducing other nodes called supernodes. It was realized that E-DEEC did not consider the proximity of the advanced nodes to the Base station in its election probabilities. This compelled the supernodes with higher residual energy but far from the Base station to dissipate a large amount of energy in conveying their reports to the Base station. In this research work, an improved heterogeneous algorithm, iE-DEEC, is proposed. The new scheme considered the distance of supernodes and the average distance of the nodes to the Base station (BS) in selecting the cluster heads (CHs). Also, different amplification energy levels have been implemented in this protocol to minimize the energy consumption during the communication between the CHs and BS and also between inter and intra cluster communication. MatLab 2017a was used for simulation to evaluate the performance of the new scheme and the existing protocol. The simulation results showed that the proposed protocol performed better than E-DEEC in terms of throughputs, residual energy and network lifetime.

Key Words: E-DEEC Protocol, Network lifetime, Residual energy, Average distance, MatLab Simulation.

\section{INTRODUCTION}

In recent times, wireless sensor network (WSN) is becoming very exciting field for research work. The network is composed of tens and thousands of sensor nodes which have data capturing and processing capabilities. The nodes can capture measurement information such as temperature, pressure, humidity and noise level in any deployment area [1]. In order to reduce energy expenditure in these network, clustering technique is usually applied. In this technique, the network is put into clusters with each cluster being manage and coordinated by elected node called the cluster head $(\mathrm{CH})$. The remaining nodes in the cluster are referred to as cluster members. The measurement data is sensed by the cluster members, relay it to the $\mathrm{CH}$ which then aggregate the data. The head then send the final report to the node called the Base station (BS)[2]. Despite the clustering technique, the nodes still spent a lot of energy in processing, transmission and reception of data from one node to the other or to the BS. So the research on cluster-based routing protocols has been intensified in both homogeneous and heterogeneous wireless sensor networks to reduce the energy consumptions in such processes. In literature, several heterogeneous routing protocols have been proposed to reduce energy depletion during data transmission.

Qing et al.[3] explained a heterogeneous routing scheme, DEEC. The protocol chooses cluster head based on the ration between residual energy of each node and the average energy of the network. The scheme uses normal and advanced nodes in its two level hierarchy but confronted with a challenge where the advanced nodes are continuously discriminated when their residual energy becomes equal to the normal nodes in the network.

Elbhiri et al.[4] presented a version of DEEC protocol for heterogeneous network. This protocol, DDEEC, suggested a better solution to the main challenge observed in DEEC scheme where the advanced nodes are punished when their residual energy is reduced to the normal nodes level. The algorithm introduced threshold residual energy value, $T h_{R E V}$ based on which the average probability of each node is determined. This gives equal opportunity to both normal and advanced nodes to become cluster heads at a point in time. 
Saini et al. [5] described another form of DEEC model, E-DEEC for heterogeneous networks by adding another nodes called super nodes to the normal and advanced nodes. This makes the scheme to have three types of sensor nodes. The super nodes have the highest residual energy compared to the other nodes. The simulation results showed that, E-DEEC outperforms DEEC in terms of stability period and network life enhancement.

Another improved version of DEEC protocol, TDEEC algorithm has been proposed by [6]. The scheme utilizes three level of nodes which are differ in their initial energy with slight changes in the probability function. The simulation results proved that, TDEEC performed better in terms stability period and network lifetime.

Authors in [7] discussed the results of the modified version of LEACH, MODLEACH for homogeneous networks. MODLEACH introduced two technique to enhance its performance which include, the efficient cluster head replacement model and different amplification energy levels. These methods were proposed firstly, to give a cluster head another opportunity to continue as cluster head in the next round provided its energy is not exhausted. Secondly, to provide different amplification levels to three types of communications that were identified; the inter and intra cluster communication and the communication between the cluster heads (CHs) and the BS. We implemented different amplification energy levels in this research work to conserve energy during inter and intra cluster communications and communication between cluster heads and the Base station.

The remainder of this research is organized as follows: Section II explained the methodology used, simulation results and analysis are discussed in Section III and conclusion is then drawn in Section IV

\section{METHODOLOGY}

In this section, the operation of both the proposed and existing protocols are explained.

\section{A. The Existing E-DEEC protocol}

The E-DEEC protocol operates similar to the DEEC scheme in terms of selecting the cluster heads based on the residual energy of each. E-DEEC however, introduced another node called super to the DEEC nodes, the advanced and normal nodes to increase the heterogeneity of the network. This makes this scheme to have three levels of nodes based on their initial energy. The scheme therefore suggested a corresponding election probability of each level of the nodes without considering the distance factor and is given by (1)

$$
P i=\left\{\begin{array}{cc}
\frac{p_{o p t} E_{i}(r)}{(1+m(a+m o . b)) \bar{E}(r)} & \text { for normal node } \\
\frac{p_{o p t}(1+a) E_{i}(r)}{(1+m(a+m o . b)) \bar{E}(r)} & \text { for advanced node } \\
\frac{p_{o p t}(1+b) E_{i}(r)}{(1+m(a+m o . b)) \bar{E}(r)} & \text { for super node }
\end{array}\right.
$$

The corresponding thresholds for the above classes of sensor nodes is given by (2)

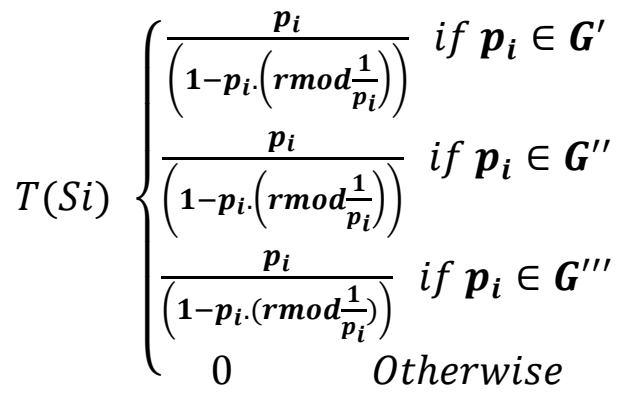

where $\boldsymbol{G}^{\prime}$ is the set of normal nodes that have not become cluster heads within the last $\frac{1}{\boldsymbol{p}_{\boldsymbol{i}}}$ rounds of the epoch $\boldsymbol{G}^{\prime \prime}$ "'is the set of advanced nodes that have not become cluster heads within the last $\frac{1}{\boldsymbol{p}_{\boldsymbol{i}}}$ rounds of the epoch and $\boldsymbol{G}^{\prime \prime \prime}$ is the set of super nodes that have not become cluster heads within the last $\frac{1}{\boldsymbol{p}_{\boldsymbol{i}}}$ rounds of the epoch 


\section{B. Proposed iE-DEEC Protocol}

The proposed protocol operates similar to E-DEEC protocol. The new scheme however modified the election probability of selecting the cluster heads among the super nodes. The new probability for choosing the cluster heads (CHs) in this new algorithm depends also on the ratio of distance of the super nodes and the average distance of all the nodes from the Base station(BS). This allowed the super nodes which have higher residual energy and also closer to the BS to have better chance of becoming cluster heads than those which may be having a high residual energy but far from the BS. This makes the super nodes which are elected as cluster heads to use less energy in relaying their report to the BS. The new election probability is given by (3)

$$
P i=\left\{\begin{array}{cc}
\frac{p_{\text {opt }} \boldsymbol{E}_{\boldsymbol{i}}(\boldsymbol{r})}{(\mathbf{1}+\boldsymbol{m}(\boldsymbol{a}+\boldsymbol{m o . b})) \overline{\boldsymbol{E}}(\boldsymbol{r})} & \text { for normal node } \\
\frac{\boldsymbol{p}_{\text {opt }}(1+a) \boldsymbol{E}_{\boldsymbol{i}}(\boldsymbol{r})}{(\mathbf{1}+\boldsymbol{m}(\boldsymbol{a}+\boldsymbol{m o . b})) \overline{\boldsymbol{E}}(\boldsymbol{r})} & \text { for advanced node } \\
\frac{\boldsymbol{p}_{\text {opt }}(1+b) \boldsymbol{E}_{\boldsymbol{i}}(\boldsymbol{r})}{(\mathbf{1}+\boldsymbol{m}(\boldsymbol{a}+\boldsymbol{m o . b})) \overline{\boldsymbol{E}}(\boldsymbol{r})} x \frac{\text { distance }(i)}{D(\text { ave })} & \text { for super node }
\end{array}\right.
$$

Also, the technique introduced by [7] has been used in this protocol to reduce energy expenditure as a result of inter and intra cluster communication and that of $\mathrm{CH}$ and $\mathrm{BS}$ and it is given by (4)

$$
\left\{\begin{array}{l}
k E_{e l e c t}+k \epsilon_{\frac{f s}{10}} d^{2}, \text { if } d>d_{1} \\
k E_{\text {elect }}+k \epsilon_{\frac{m p}{10}} d^{4}, \text { if } d \leq d_{1}
\end{array}\right.
$$

Where $\boldsymbol{d}=\sqrt{\frac{\mathrm{Efs}}{\mathrm{Emp}}}$ and $\boldsymbol{d}_{\mathbf{1}}=\sqrt{\frac{\text { Efs1 }}{\mathrm{Emp} 1}}$ given that:

$\mathrm{Efs} 1=\frac{\mathrm{fs}}{10} \quad$ and $\quad \operatorname{Emp} 1=\frac{\mathrm{mp}}{10}$

\section{Simulation Results and Analysis}

To evaluate the effectiveness of our iE-DEEC protocol and existing E-DEEC algorithm, we used MatLab 2017a for simulation. In this experiment, a random network of 100 nodes is used in $100 \mathrm{~m} \times 100 \mathrm{~m}$ square area and the Base station was placed at the middle of the deployment area $(50 \mathrm{~m}, 50 \mathrm{~m})$ as in DDEEC scheme. $20 \%$ advanced nodes and $30 \%$ super nodes which have 1.5 times and 3 times more energy respectively than the normal nodes were deployed $(\mathrm{m}=0.5, \mathrm{mo}=0.4, \mathrm{a}=1.5, \mathrm{~b}=3)$. Other parameters used in the simulation are shown in the Table1.

Table I: Simulation Parameters

\begin{tabular}{|l|l|l|}
\hline $\mathbf{S} / \mathbf{N}$ & Parameter & Values \\
\hline $\mathbf{1}$ & $E_{\text {elect }}$ & $50 \mathrm{~nJ} / \mathrm{bit}$ \\
\hline $\mathbf{2}$ & $E_{f s}$ & $10 \mathrm{pJ} / \mathrm{bit} / \mathrm{m}^{2}$ \\
\hline $\mathbf{3}$ & $E_{m p}$ & $0.0013 \mathrm{pJ} / \mathrm{bit}^{2} \mathrm{~m}^{2}$ \\
\hline $\mathbf{4}$ & $E_{0}$ & $0.5 \mathrm{~J}$ \\
\hline $\mathbf{5}$ & Message size, $\quad k$ & 4000 \\
\hline $\mathbf{6}$ & $n$ & 100 \\
\hline $\mathbf{7}$ & $p_{o p t}$ & 0.1 \\
\hline & $E_{D A}$ & $5 \mathrm{~nJ} / \mathrm{bit} / \mathrm{message}$ \\
\hline
\end{tabular}

Figure. 1 shows the number of alive nodes during each round of the simulation procedure for the iE-DEEC and existing routing protocol, E-DEEC. From the graph, it can be noticed that, the lifetime of the network has been enhanced appreciably in iE-DEEC compared to E-DEEC. Nodes stay alive up to 1520 rounds in E-DEEC while remain alive up to 2500 rounds in iE-DEEC. This shows that, nodes stayed alive for lengthier time in iE-DEEC than the existing scheme and hence better lifetime than E-DEEC routing protocol. The longer lifetime of the new algorithm is as a result of the energy conserving techniques that was implemented in the protocol. Firstly, distance factor reduces the energy drainage of the distant super nodes because they have less chance of becoming cluster heads. So their energy is conserved. Secondly, the different amplification energy levels applied in this scheme also controlled the energy consumption during inter and intra cluster communication. 


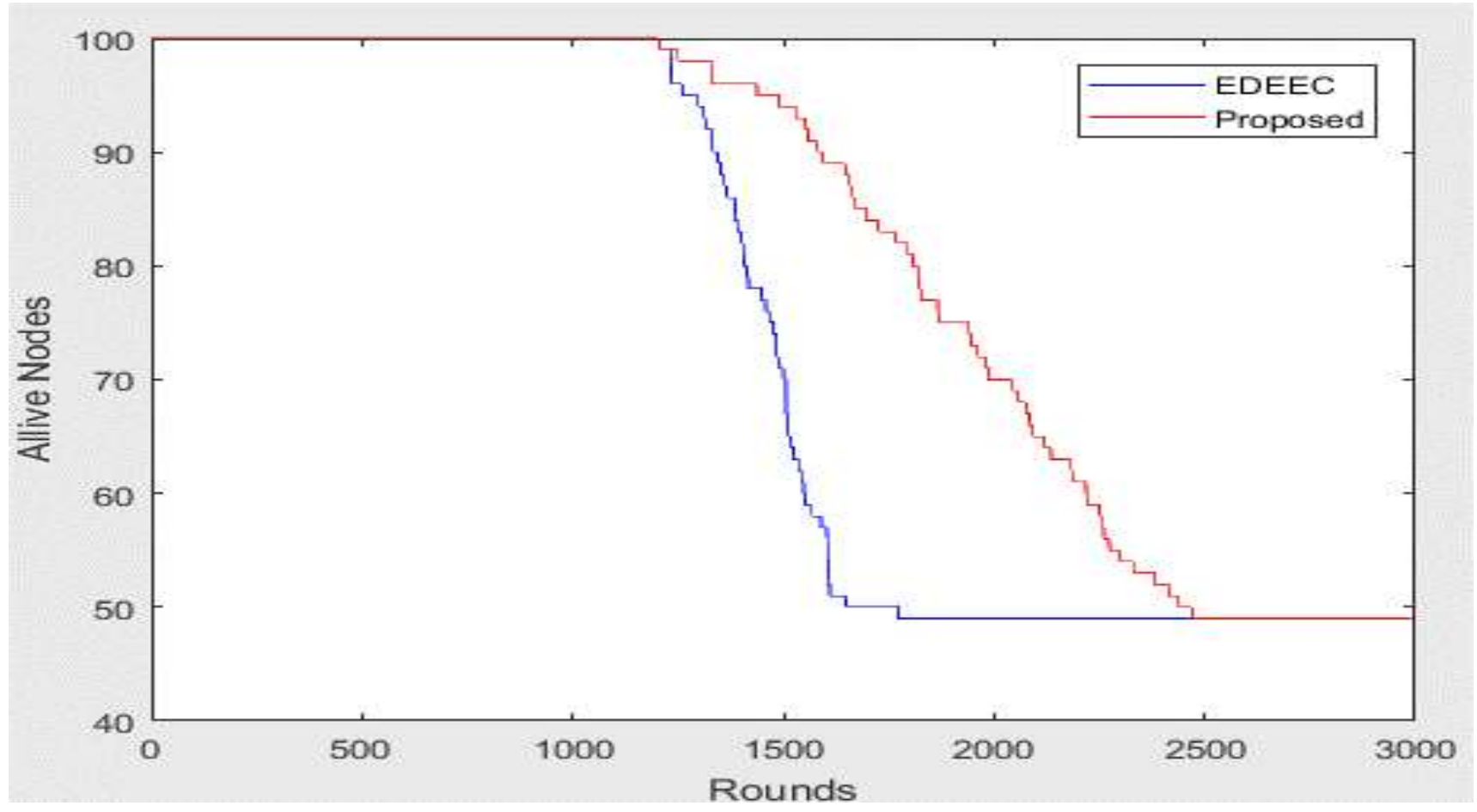

Figure. 1: Number of the Alive nodes per round

Furthermore, it was noticed that, the death rates in iE-DEEC is lower compare to that of iE-DEEC as seen in Figure 1. This again shows that the proposed scheme has effectively reduced the number of dead nodes hence its better network lifetime.

Figure 2 also shows the quantity of data sent to the BS per round in both iE-DEEC and the existing protocols. It can be observed that, the magnitude of data sent to the BS by the existing protocol increases from 0 to less than 200000 at the end of the simulation period. Thus sending relatively small amount of data to the BS. In the new scheme, large amount of data was conveyed to the BS which is more than 200000. This performance is again as result of the distance and average distance factor that were considered in electing the super nodes as cluster heads. The super nodes that were closer to the BS have elected as the Cluster heads hence less energy is used to relay their data to the BS. So they transmit more data with less energy expenditure.

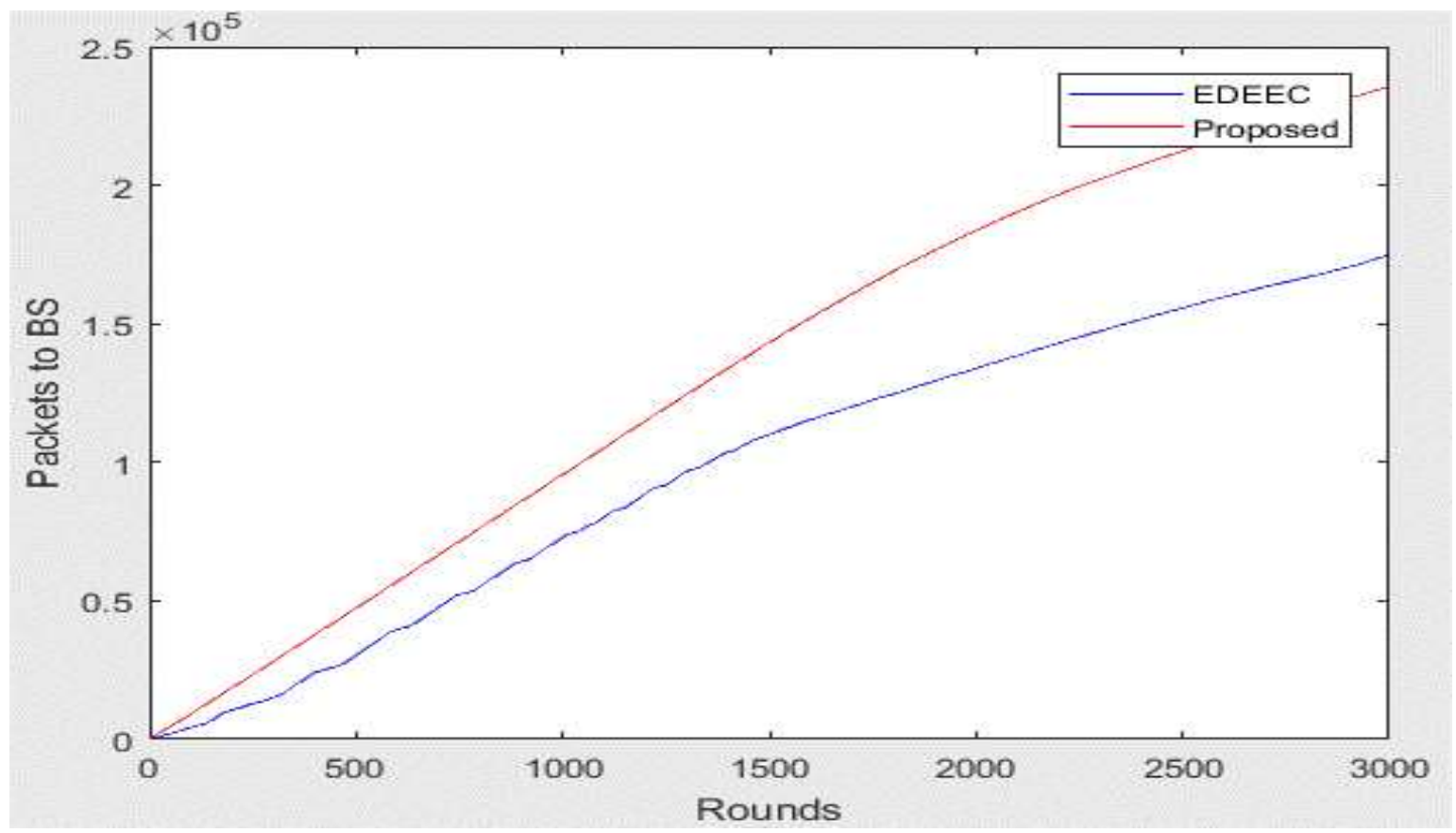

Figure 2: Number of packet to the BS per rounds

Figure 3 shows energy dissipation of the network in both routing protocols. Though the two protocols decreased linearly, E-DEEC drained its energy at approximately 1500 rounds whereas the iE-DEEC algorithm on the other hand exceeded 1700 rounds before its energy was exhausted as seen Figure 3. This indicates that iE-DEEC consumes less energy in forwarding its data to the BS. 


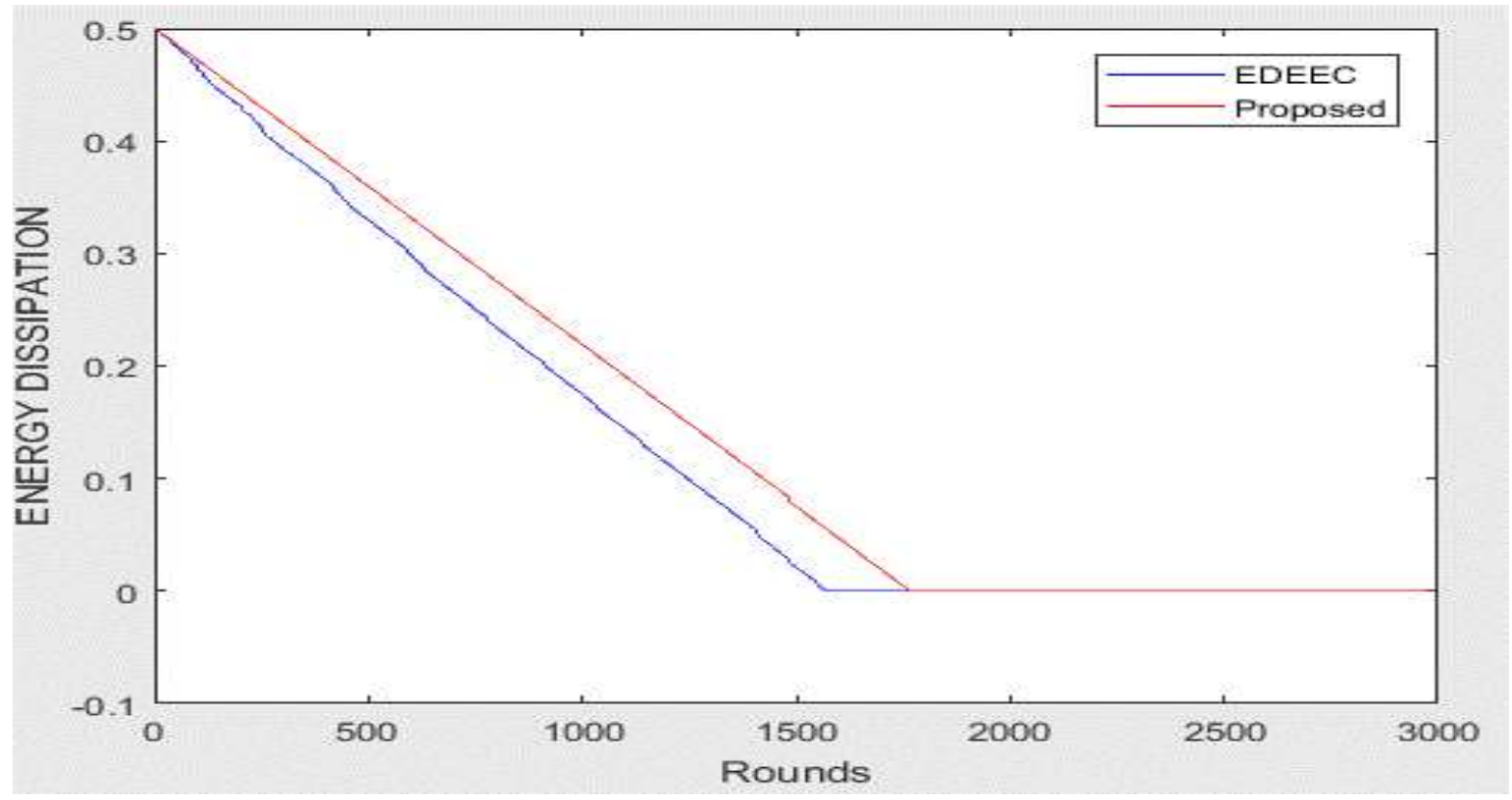

Figure 3: Remaining Energy per round

\section{CONCLUSION}

In this article, a new version of E-DEEC protocol called iE-DEEC is proposed. In this new algorithm, the election probability of the super nodes has been redesigned to consider both the distance of the super nodes and the average distance of all the nodes from the Base station. The new protocol makes the super nodes with high residual energy and also nearer the BS to have better chance of becoming cluster heads. Furthermore, the protocol implement different application energy levels which has reduced the inter and intra cluster communication and also, the communication between the cluster heads and the Base station. All these, have indeed minimized the energy consumption in these nodes which resulted into better throughputs, residual energy and longer lifetime of the network.

\section{REFERENCES}

[1] D. Estrin, R. Govindan, J. Heidemann et al., "Next century challenges: Scalable coordination in sensor networks." pp. 263 270 .

[2] H. Dhawan S. Waraich,” A Comparative Study on LEACH Routing Protocol and its Variants in Wireless Sensor Networks: A Survey”, International Journal of Computer Applications (0975 - 8887) Vol. 95- No.8, 2014, pp.21-27.

[3] L. Qing, Q. Zhu, and M. Wang, "Design of a distributed energy-efficient clustering algorithm for heterogeneous wireless sensor networks", Computer Communications, vol. 29, no.12, 2006, pp.2230-2237.

[4] B. Elbhiri, R, ,Saadane, S. El Fkihiand and D. Aboutajdine, "Developed Distributed Energy-Efficient Clustering (DDEEC) for heterogeneous wireless sensor networks", I/V Communications and Mobile Network (ISVC), 5th International Symposium on, vol., no., 2010, pp.1-4

[5] P. Saini and A. K. Sharma, "E-DEEC- Enhanced Distributed Energy Efficient Clustering Scheme for heterogeneous WSN", 1st International Conference on Parallel, Distributed and Grid Computing,2010, pp. 205-210

[6] P. Saini, and A. K. Sharma, “Energy Efficient Scheme for Clustering Protocol Prolonging the Lifetime of Heterogeneous Wireless Sensor Networks”, International Journal of Computer Applications 6(2),2010, pp. 30-36.

[7] D. Mahmood, N. Javaid,, S. Mehmood, S. Qureshi, A.M. Memon., and , T. Zaman,” MODLEACH: a variant of LEACH for WSNs"26th IEEE Canadian Conference on Electrical and Computer Engineering (CCECE2013)", Regina, Saskatchewan, Canada, 2013, pp. 1-6. 
International Journal of Engineering Research And Advanced Technology, Vol.5, Issue 1, January-2019 